\title{
Improvement of Image Matching Method based on Gray Value and Contracting Mapping Principle
}

\author{
Yuncong Sun ${ }^{1, a}$, Zongshun $\mathrm{Hu}^{2, \mathrm{~b}}$ \\ ${ }^{1}$ Department of Aviation Ammunition,Air Force Logistics College,Xuzhou 221000,China \\ ${ }^{2}$ Department of Four Station,Air Force Logistics College,Xuzhou 221000,China \\ asnailclan@hotmail.com, bfsjobs.hu@icloud.com
}

Key words: gray value; contracting mapping principle; fixed point

\begin{abstract}
General image matching method is faced with many problems like the selection of template and mismatching. In order to solve these problems, in this paper, general image matching method is improved based on contracting mapping principle, matching is implemented by finding the fixed point between images. The validity of the method is verified through experiments. The result shows that the improved method has higher accuracy, can avoid mismatching problem occurring in general method.
\end{abstract}

\section{Introduction}

In the process of computer vision recognition, it is often necessary to compare two or more images obtained by different sensors or the same sensor at the same time and under different imaging conditions to find the common scene in the group of images, or is based on the known pattern to another map to find the appropriate model, which is called image matching [1]. In general, due to different time, different sensors, different viewing angle of imaging conditions are different, so even for the same object, the image shown in the geometric characteristics, optical properties, spatial location will be very different, and noise And other interference will make the image very different, image matching is through these differences to find their similarities.

Image matching is also called scene matching or image matching [2]. The method is divided into two categories: one is based on gray correlation [3-5]; the other is feature-based matching [6-7]. The former mainly uses the one-dimensional or two-dimensional sliding template in the spatial domain for image matching. The difference between the different algorithms is mainly reflected in the selection of templates and related criteria. Generally, this method has high matching rate, but the computation is large and slow. The matching feature can be used as the matching unit, and the matching speed is fast, but the matching precision is not high. Based on the principle of contraction mapping, this paper proposes an improved matching method based on the traditional gray-level matching method. By matching the fixed points between the images, the feasibility of the method is proved by an example. High accuracy, can effectively avoid the phenomenon of mismatch and complex matching template selection process.

\section{Gray matching principle}

The general gray-level matching method is the normalized gray-level matching method. The basic principle is that a gray-scale matrix of a real-time image window with a certain size is matched with all the possible gray- According to a similarity measurement method for search and comparison [8].

In the case of an 8-bit image, the template $t$ ( $m \times n$ pixels) are translated on the search graph $s$ (sHeight $\times$ sWidth pixels) (Fig.1). The sub-blocks covered by the template are called the search subgraphs $s_{i j}, i, j$ is the position of the subgraph in the search graph $s$, the searchable range of the template on the search graph is

$$
1 \leq i \leq \text { sHeight }-m
$$


$1 \leq j \leq$ sWidth $-n$

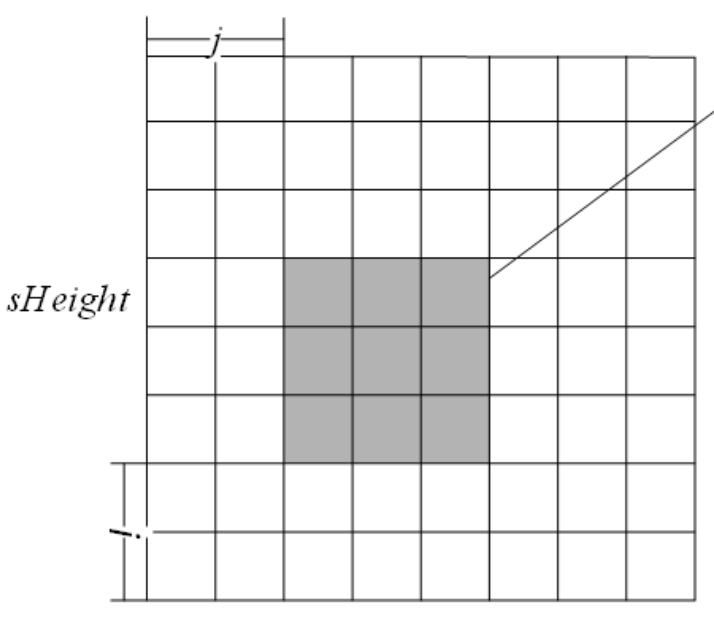

Search for

sub-graphs

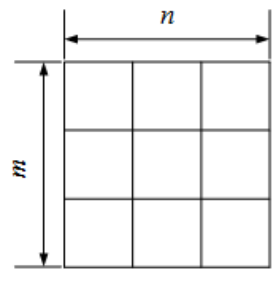

Template

Fig.1 Search graph $s$ and template $t$

By comparing the similarity between the template $t$ and the search subgraph $s_{i j}$, the matching process is completed. The similarity is measured as follows:

$$
\begin{gathered}
D(i, j)=\sum_{m=1}^{M} \sum_{n=1}^{N}\left[s_{i j}(m, n)-t(m, n)\right]^{2} \\
=\sum_{m=1}^{M} \sum_{n=1}^{N}\left[s_{i j}(m, n)\right]^{2}-2 \sum_{m=1}^{M} \sum_{n=1}^{N} s_{i j}(m, n) \times t(m, n)+\sum_{m=1}^{M} \sum_{n=1}^{N}[t(m, n)]^{2}
\end{gathered}
$$

The first term of the expansion is the energy of the subgraph, the last term is the energy of the template, and the middle term is the cross-correlation coefficient of the template and the subgraph, which varies with $(i, j)$. When the template and sub-map to achieve matching, the intermediate term has a maximum value, its normalized, can be template matching the correlation coefficient:

$$
r(i, j)=\frac{\sum_{m=1}^{M} \sum_{n=1}^{N} S_{i j}(m, n) \times t(m, n)}{\sqrt{\sum_{m=1}^{M} \sum_{n=1}^{N}\left[S_{i j}(m, n)\right]^{2}} \sqrt{\sum_{m=1}^{M} \sum_{n=1}^{N}[t(m, n)]^{2}}}
$$

When the template and the search sub-map exactly the same, the correlation coefficient $(i, j)$ value is 1 , the general matching algorithm is to row-by-row search for each search subgraph to find the maximum correlation coefficient $r_{\max }$, and then Find the corresponding sub-map is to match the target. Another algorithm is to measure the error of $t$ and $s_{i j}$, the calculation method is

$$
E(i, j)=\sum_{m=1}^{M} \sum_{n=1}^{N}\left|s_{i j}(m, n)-t(m, n)\right|
$$

$E(i, j)$ minimum is the matching target. In order to improve the speed, take the error $E_{0}$ threshold, when $E(i, j)>E_{0}$ to stop the calculation, and go to the next area to continue the calculation.

\section{Improvement method}

\subsection{Compression mapping principle}

The principle of contraction mapping, also known as the Banach fixed point principle, is an important tool of metric space theory, which guarantees the existence and uniqueness of the fixed points of metric space self-mapping and provides the solution of these fixed points Constructive method [9].

Definition 1 Given a metric space $(X, \rho)$ and a mapping $T: X \rightarrow X$, if $x \in X$ is present, 


$$
T x=x
$$

$x$ is the fixed point of mapping $T$. If there is a positive constant $q \in(0 / 1)$, so that any given $x y=X$ is true

$$
\rho(T x, T y) \leq q \rho(x, y)
$$

Then $T$ is a compression map on $X$.

Theorem 1 (contraction mapping principle) Let $(X \not \rho)$ be a complete metric space and $T \not X \rightarrow X$ is a contractive map, and then the mapping $T$ has only one fixed point.

Prove The contraction mapping is continuous, so for any convergent point column

$$
x_{n} \rightarrow x_{0}(n \rightarrow \infty)
$$

There must be

$$
T x_{n} \rightarrow T x_{0}(n \rightarrow \infty)
$$

In fact, we base

$$
\rho\left(T x_{n}, T x_{0}\right) \leq q \rho\left(x_{n}, x_{0}\right)
$$

We can see

$$
\rho\left(x_{n}, x_{0}\right) \rightarrow 0(n \rightarrow \infty)
$$

So there

\subsection{Algorithmic flow}

$$
\rho\left(T x_{n}, T x_{0}\right) \rightarrow 0(n \rightarrow \infty)
$$

In this paper, based on the principle of compression mapping to improve the general matching method, without matching template selection, but will need to match the two images overlap together, as shown in Fig.2, if not overlap can be achieved through stretching. In the corresponding position of image 1 and image 2, a rectangular region $A_{i j}$ and $B_{i j}$ is selected as the search subgraph, and $A_{i j}$ and $B_{i j}$ are gray-matched to solve the correlation coefficient. This completes a matching. And then follow the general matching method in the template traversal process to complete the entire match. Among them, the maximum correlation coefficient or the smallest error of the two regions is the fixed point of the two maps, that is, two images in the same location on behalf of the same scene. In order to complet the matching of the two images.

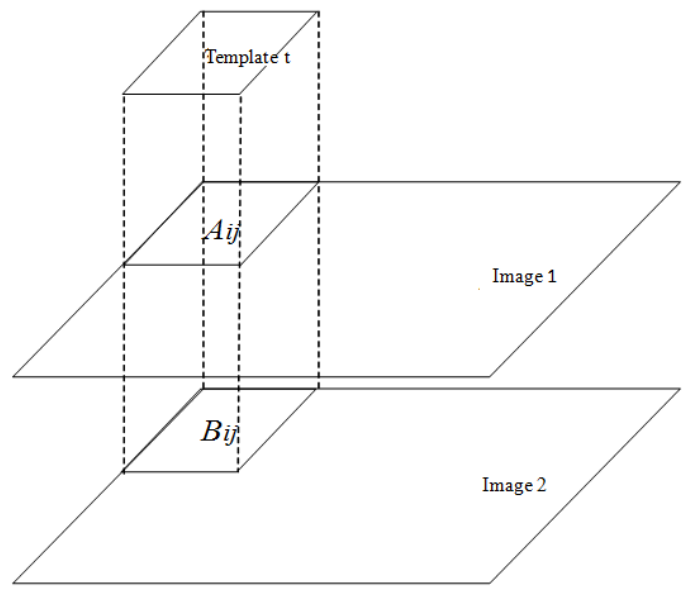

Fig.2 Matching diagram

In accordance with Fig. 2, the specific process can be described as:

Step 1: Select rectangle areas $A_{i j}$ and $B_{i j}$ of the size as the search subgraphs at the corresponding positions of image 1 and image 2 .

Step 2: Calculate the correlation coefficients of search subgraphs $A_{i j}$ and $B_{i j}$, and calculate the difference $E_{i j}$. 
Step 3: If $E_{i j}$ is greater than $E_{0}$, the calculation of the next search subgraph is transferred. If $E_{i j}$ is less than $E_{0}$, then counter $N$ (used to calculate the number of $E_{i j}$ less than $E_{0}$ ) plus 1, and note the location, set to re-search points.

Step 4: Perform a detailed search on the re-searchable points, calculate the $E_{i j}$ of the resulting points, and note the location of the smallest $E_{i j}$.

Step 5: Output the coordinates of the fixed point and the correlation coefficient.

\section{Simulation experiment and analysis}

In order to verify the effectiveness of the new algorithm, the experiment uses two photos of the waterfall landscape at different angles as the search map, and the matching is realized in the MATLAB environment. Image size is $2200 \times 1200$, theoretically, the smaller the search subgraph the higher the accuracy, but the matching time will be consumed longer, here we use three kinds of search sub-map size matching, the size of $60 \times 60,70 \times 70,80 \times 80$. Select the search sub-map for the $80 \times 80$ when the match, the results shown in Fig.3.

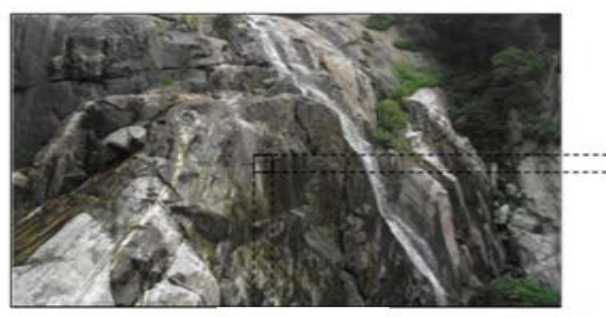

Image 1

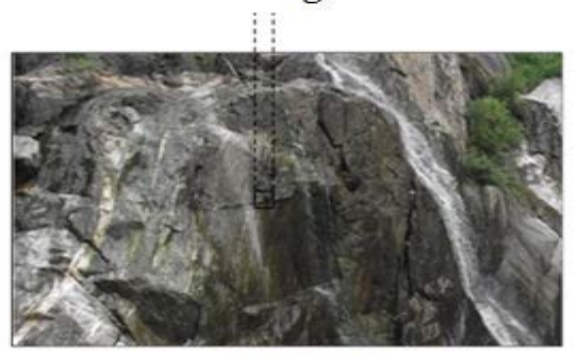

Image 3

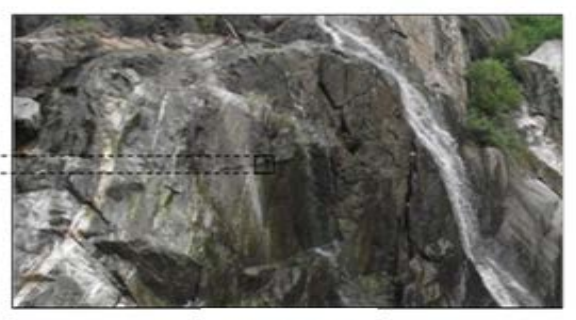

Image 2

Fig.3 Search sub-graph of $80 \times 80$ when the matching effect map

The square box represents the fixed point of the match. As can be seen from the figure, the fixed point has the same abscissa and ordinate in the image 1 and the image 2, and the same scene is reflected in the photograph. The results of the three matches are compared to obtain the following table.

Table 1 Comparison of matching results under different search subgraphs

\begin{tabular}{ccc}
\hline $\begin{array}{c}\text { Search for submap } \\
\text { dimensions }\end{array}$ & Matching time / s & Correlation coefficient \\
\hline $60 \times 60$ & 27.4 & 0.986 \\
$70 \times 70$ & 18.8 & 0.978 \\
$80 \times 80$ & 10.6 & 0.971 \\
\hline
\end{tabular}

It can be seen from the data in the table that as the search subgraph grows from small to large, its matching time gradually decreases, but its correlation coefficient decreases. The reason is that the increase of the search subgraph reduces the possible matching points in the image, but since the fixed point is only a small region in the image, increasing the search subgraph will inevitably lead to the decrease of the correlation coefficient. 


\section{Conclusion}

Compared with the original method, the improved method has the advantage of being able to search for every point in the image, and has strong anti-noise ability without the need of selecting the template in advance, thus avoiding the problem of low matching accuracy due to improper template selection. The relative coefficients of the fixed region are relatively large. When there are many matching points, the fixed points can be easily distinguished, which avoids the problem of mismatch. The shortcoming is that the smaller the precision of search subgraph is, the higher the precision is, but the unavoidable increase of matching time. How to get the best result between accuracy and time is still under further study.

\section{Reference}

[1] Lisa. Gottesfeld Brown. A Survey of Image Registration Techniques.Department of Computer Science,Columbia University, New York, NY 10027, 1992.

[2] Zuxun Zhang, Jianqing Zhang. Digital photogrammetry[M]. Wuhan: Wuhan University of Science and Technology Press, 1996.

[3] Qiang LI, Bo Zhang.A fast matching algorithm based on image gray level [J] .Journal of Software, 2006, Vol.17: 216-222

[4] Lijie Fan, Yanfei Wang, Xin Gao.A New Image Matching Method Based on Gray Scale [J] .Computer Information, 2007, 23 (10): 296-297

[5] Zhiming Sang. Several gray-based image matching algorithm research [D]. Nankai University, 2011

[6] Xiaoying Yang, Yali Wang, Lei Wang.An Image Matching Algorithm Based on Feature Point Set [J] .Computer \& Network, 2009, 17: 63-68

[7] Jing Hou, Mengxin Li, Languang Zhao.Image Matching Method Based on Feature and Region [J] .Shenyang Jianzhu University (Natural Science Edition), 2012, 28 (2): 380-384

[8] Huizhen Ding, Shaoguang Zhou.An Improved Gray Matching Algorithm [J] .Resources, Environment \& Engineering, 2006, 20 (1): 52-55

[9] Zhiying Deng, Jianhui Pan. Banach fixed point theorem and its application [J]. Advanced Mathematics Research, 2013, Vol 16 No. 4: 78-80 